\title{
Association of clinical characteristics of patients presenting with influenza like illness or severe acute respiratory illness with development of acute respiratory distress syndrome
}

\author{
Muhammad ljaz', Muhammad Jaffar Khan², Jawad Khan³, Usama² \\ 1 Northwest General Hospital Peshawar, Khyber Pakhtunkhwa \\ 2 Institute of Basic Medical Sciences, Khyber Medical University Peshawar, Khyber Pakhtunkhwa \\ 3 Department of Pulmonology, Government Lady Reading Hospital, Khyber Pakhtunkhwa, Pakistan
}

\begin{abstract}
Clinical judgement and suspicion of influenza based on signs and symptoms of influenza-like illness and severe acute respiratory illness are critical for better patient outcome. Whether clinical characteristics of patients are associated with the development of acute respiratory distress syndrome was the aim of this study. We included all patients $(\mathrm{n}=37)$ presenting with influenza like illness (ILI) or severe acute respiratory illness (SARI) to a tertiary care hospital in northwest Pakistan during December 2015 until the end of January 2016. Each patient was assessed for signs and symptoms, clinical features, treatment, complications and outcome of ILI and SARI. Throat or nasopharyngeal swabs were obtained from 36 patient and analyzed for the presence of Influenza virus by quantitative PCR. Patients presenting with ILI or SARI were febrile $(\mathrm{p}<0.001$, one sample $t$-test), significantly tachypneic $(\mathrm{p}<0.001)$ and had critically lower oxygen saturation $(\mathrm{p}<0.001)$. Nasal congestion at presentation ( $p=0.006$, chi-square test for association) and infiltrates on chest radiographs $(p=0.025)$ were significantly associated with acute respiratory distress syndrome. Likelihood of the occurrence of ARDS was significantly increased with decrease in oxygen saturation (Odds ratio; $0.75,95 \% \mathrm{CI} ; 0.46,1.21, \mathrm{p}=0.048$ ) and marginally significantly increased in lower age (Odds ratio; $0.82,95 \% \mathrm{CI} ; 0.58,1.15, \mathrm{p}=0.055)$ and higher white cell count (Odds ratio; $1.001,95 \% \mathrm{CI}$; 0.99, 1.002, $\mathrm{p}=0.054$ ).
\end{abstract}

Corresponding author: Dr. Muhammad Jaffar Khan, Institute of Basic Medical Sciences, Khyber Medical University Peshawar, Khyber Pakhtunkhwa 25000, Pakistan. Tel. +92.333.9369166.

E-mail: jaffar.khan@kmu.edu.pk

Key words: Influenza-like illness; severe acute respiratory illness; influenza; acute respiratory distress syndrome.

Received for publication: 19 August 2016

Accepted for publication: 1 January 2017

(C) Copyright M. Ijaz et al., 2017

Tipografia PI-ME Editrice, Italy

Monaldi Archives for Chest Disease 2017; 87:765

doi: $10.4081 /$ monaldi.2017.765

This article is distributed under the terms of the Creative Commons Attribution Noncommercial License (by-nc 4.0) which permits any noncommercial use, distribution, and reproduction in any medium, provided the original author(s) and source are credited.
The presence of Influenza type A/H1N1pdm09 strains was confirmed in $7 / 11$ patients. However no significant difference was observed in the clinical features and complications of PCR positive and negative patients. Clinical signs and symptoms of influenza-like illness or severe acute respiratory illness significantly predict the development of complications irrespective of the positivity or negativity of laboratory qPCR reports.

\section{Introduction}

Influenza, commonly known as flu, is a highly contagious acute respiratory viral disease. The spectrum of disease range from mild to severe illness and even death. Almost every year, seasonal flu epidemics are mainly caused by human Influenza A and B viruses [1]. In recent years, a global outbreak of H1N1 swine flu took its origin from a pig farm in Mexico in April 2009 [2], gradually spreading towards Southern hemisphere and underdeveloped regions of the world causing laboratory confirmed cases of pandemic influenza H1N1 pdm/09 in 209 countries and causing a death toll around 17,000 people [2]. Recently, as of February 2016, more than 250 laboratory confirmed H1N1 pdm/09 cases have been reported from Caribbean islands and South America [3]. Out of more than 155 thousand specimens collected from 98 countries by World Health Organization in the month of February 2016, approximately $30 \%$ tested positive for influenza viruses, of which $80 \%$ were influenza A, mainly (H1N1)pdm/09 [3].

Patients with H1N1 generally present with influenza like illness (ILI; as defined by WHO as those with sudden onset of fever $\left(>38^{\circ} \mathrm{C}\right)$ and cough/sore throat within seven days of onset) or severe acute respiratory illness (SARI; defined as patients with sudden onset of fever $\left(>38^{\circ} \mathrm{C}\right)$, cough/sore throat that required hospital admission within 7 days) [4]. Since the detection of first laboratory confirmed case of swine flu in Pakistan on August $10^{\text {th }} 2009$ [5], sporadic cases are reported on yearly basis. This winter 2015-2016 the country had an epidemic of H1N1 flu with more than 240 cases confirmed until February 2016 [6]. In Pakistan, clinical specimens obtained from patients presenting with ILI or SARI are usually reported after 10-12 days for logistic reasons and lack of facilities for quantitative determination of Influenza virus in the city. On the other hand, the infectivity period of patient presenting with these symptoms is from one day before the onset of symptoms and until 3 days after the onset of symptoms [7]. Hence, isolation and definitive treatment of patient after qPCR confirmation is expected to significantly increase the morbidity, mortality and cross-infection. In this scenario, clinical judgement and suspicion is critical for the better primary and secondary prevention of this disease. Therefore, the aim of this study was to evaluate the magnitude and clinical characteristics of patients presenting with influenza like illness or severe acute respiratory illness admitted to tertiary care hospitals in northwest Pakistan between December 2015 and February 2016. 


\section{Materials and Methods}

\section{Study design}

This was a prospective observational study of all 37 patients (females, $\mathrm{n}=25$, males; $\mathrm{n}=12$ ) presenting with severe acute respiratory illness (SARI) or Influenza like illness (ILI) to a tertiary care hospital (Government Lady Reading Hospital) in Peshawar Khyber Pakhtunkhwa Pakistan between December 2015 until the end of January 2016. Initially patients presented to various departments (including Pulmonology, Medicine, and intensive care), they were eventually isolated in Pulmonology department for further management.

\section{Methods}

Each patient was assessed according to the standard protocols issued by the National Institute of Health (NIH) Islamabad. Briefly, each patient was assessed for signs and symptoms of ILI and SARI, history of contact with sources of influenza, clinical complications, and outcomes of disease during the process of treatment in hospital. Throat or nasopharyngeal swabs were obtained from 36 patients and sent to $\mathrm{NIH}$ central laboratory Islamabad for quantitative PCR for Influenza virus. All patients received standard hospital care as per protocols. Informed oral consent was obtained from each patient. The study was approved by the Ethics committee of Khyber Medical University Peshawar and Government Lady Reading Hospital Peshawar.

\section{Analysis}

Data was organized in Microsoft Excel 2013 (Microsoft ${ }^{\circledR}$ ) and then analyzed using Minitab ver. 17 (Minitab® Inc., State College, PA, USA). Continuous data was expressed as mean and standard deviation, while categorical data was expressed as counts and percentage. One sample t-test was used to calculate the difference between body temperature, respiratory rate, oxygen saturation, and white cell count and their standard cut-off values. Binary logistic regression analysis was used to assess the association of the occurrence of acute respiratory distress syndrome with age, respiratory rate, body temperature, white cell count, and oxygen saturation. P-value of less than 0.05 was considered significant.

\section{Results}

All patients $(n=37)$ with mean $( \pm$ SD) age of $40.46( \pm 15.27)$ years presenting with influenza like illness (ILI) or severe acute respiratory illness (SARI) were included in this study. Of these 25 (67.57\%) were females and $12(32.43 \%)$ were males. Most of these patients were from Khyber Pakhtunkhwa [25 (67.57\%)], while the rest were belonging to federally administered tribal areas (11/37) and Afghanistan (1/37). All women presenting with symptoms were house wives [24 (64.8\%)], the rest included drivers [3 (8.18\%)], students [2 (5.41)], painter, security guard and shopkeeper [1 (2.7\%) each], and other professions [3 (13.5\%)]. Most of the patients were inpatients (35/37, 94.59\%) presenting to pulmonology [31 (86.11)], Medicine [2 (5.55\%)], or other departments such as intensive care unit, high dependency unit or other wards [4 (11.11\%)].

Although most of the patients presented with cough 36(97.3\%) and fever (temperature $>38^{\circ} \mathrm{C}$ ) [32(86.48\%)], index of suspicion of an influenza infection was very low [1 (2.7\%)]. Based on the standard criteria for SARI case definition, $86 \%$ patients were labeled as having severe acute respiratory illness. Very few patients [3 (8.11\%)] gave a history of contact with probable sources of influenza.

Apart from fever and cough, patients mainly presented with shortness of breath, sore throat, abnormal breath sounds, wheezing and nasal congestion (Table 1). Patients presenting with ILI or SARI were

Table 1. Clinical characteristics of patients presenting with influenza like illness or severe acute respiratory illness.

\begin{tabular}{|c|c|c|c|c|}
\hline Characteristic & $\begin{array}{l}\text { Total } \\
\text { N (\%) }\end{array}$ & $\begin{array}{c}\text { Developed ARDS } \\
\text { N }(\%)\end{array}$ & $\begin{array}{c}\text { No ARDS } \\
\text { N }(\%)\end{array}$ & p-value* \\
\hline \multicolumn{5}{|l|}{ Findings on chest radiograph } \\
\hline Normal chest X-ray & $5(13.51)$ & $1(20)$ & $4(80)$ & 0.508 \\
\hline Infiltrate & $22(59.46)$ & $4(18.18)$ & $18(81.82)$ & 0.025 \\
\hline Consolidation & $27(72.97)$ & $10(37.04)$ & $17(36.96)$ & 0.312 \\
\hline Cavitations & $21(56.76)$ & $7(33.33)$ & $14(66.66)$ & 0.893 \\
\hline Opacities & $24(64.86)$ & $9(37.5)$ & $15(62.5)$ & 0.363 \\
\hline Effusion & $1(2.70)$ & $0(0.00)$ & $1(100)$ & $\mathrm{NC}$ \\
\hline Pneumatocele & $2(5.41)$ & $0(0.00)$ & $2(100)$ & $\mathrm{NC}$ \\
\hline Bronchopneumonia patches & $11(29.73)$ & $6(54.54)$ & $5(45.45)$ & 0.066 \\
\hline \multicolumn{5}{|l|}{ Clinical signs and symptoms } \\
\hline Cough & $36(97.3)$ & $11(91.67)$ & $1(8.33)$ & $\mathrm{NC}$ \\
\hline Fever & $36(97.3)$ & 11(91.67) & $1(8.33)$ & $\mathrm{NC}$ \\
\hline Nasal congestion & $24(64.86)$ & $4(16.66)$ & $20(83.33)$ & 0.006 \\
\hline Sore throat & $30(81.08)$ & $9(30)$ & $21(70)$ & 0.520 \\
\hline Wheezing & $28(75.68)$ & $7(25)$ & $21(75)$ & 0.096 \\
\hline Tachypnea & $10(27.03)$ & $5(50)$ & $5(50)$ & 0.173 \\
\hline Abnormal breath sounds & $30(81.08)$ & $9(30)$ & 21(70) & 0.520 \\
\hline Hemoptysis & $5(13.51)$ & $2(40)$ & $3(60)$ & 0.702 \\
\hline Sputum production & $13(35.14)$ & $4(30.76)$ & $9(69.24)$ & 0.873 \\
\hline Chest pain & $30(81.08)$ & $9(30)$ & $21(70)$ & 0.513 \\
\hline Shortness of breath & $32(86.49)$ & $10(31.25)$ & $22(68.57)$ & 0.702 \\
\hline Index of suspicion of avian influenza & $1(2.70)$ & $1(8.33)$ & $0(0.00)$ & $\mathrm{NC}$ \\
\hline History of contact with probable sources of influenza & $3(8.11)$ & $2(16.66)$ & $1(8.33)$ & $\mathrm{NC}$ \\
\hline
\end{tabular}

${ }^{*} \chi^{2}$-test for association; ARDS: acute respiratory distress syndrome; NC: value not computable due to low number. 
febrile ( $\mathrm{p}<0.001$, one sample $t$-test), tachypneic ( $\mathrm{p}<0.001$, one sample $t$-test) and had critically lower oxygen saturation $(\mathrm{p}<0.001$, one sample $t$-test) (Table 2). We performed Chi-square test to assess whether a particular mode of clinical presentation was associated with the development of ARDS and observed that nasal congestion $(p=0.006)$ was associated with the subsequent development of ARDS. Antibiotics were used by 32 (86.24\%) patients prior to admission, however use of antivirals was not reported by any of the patient. Significantly higher number of patients who did not develop ARDS used antibiotics prior to their presentation (No ARDS; 24/25 vs ARDS; 8/12, $\mathrm{p}=0.018, \chi^{2}$-test). Chest radiograph showed abnormal findings in 32 (86.49\%) patients, mainly consolidations, opacities, infiltrations or cavitations (Table 1). Infiltration seen on chest X-ray was significantly associated with the development of ARDS ( $\mathrm{p}=0.025, \chi^{2}$-test). Majority of patients [32 (86.49\%)] developed one or more than one complications as a result of the disease, of which 28 (75.68\%) patients developed pneumonia, 12(32.43\%) patients developed ARDS and 2(5.41\%) patients eventually progressed to respiratory failure. Presence of cough ( $\mathrm{p}=0.038$, Fisher's exact test), nasal congestion ( $\mathrm{p}=0.023, \chi^{2-}$ test), wheezing ( $\mathrm{p}=0.012, \chi^{2}$-test), and chest pain ( $\mathrm{p}=0.001, \chi^{2}$-test) was significantly associated with the occurrence of pneumonia. 0xygen therapy was required for 29 (78.38\%) patients and 12 (32.43\%) patients required ventilation. Three patients (8.11\%) died of the complications and the rest were discharged.

Whether the occurrence of acute respiratory distress syndrome is associated with age, temperature, respiratory rate, oxygen saturation and white cell count, binary logistic regression analysis was used considering the occurrence of ARDS as an event (Table 3). Likelihood of the occurrence of ARDS was significantly increased with a decrease in

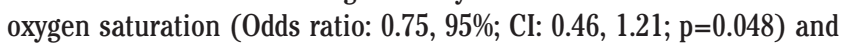
marginally significantly increased with decreasing age (Odds ratio: 0.82, 95\%; CI: 0.58, 1.15; $\mathrm{p}=0.055$ ) and higher white cell count (Odds ratio: $1.001,95 \%$; CI: 0.99, 1.002; $\mathrm{p}=0.054)$.

Based on the presentation of patients, throat swabs were obtained from 34 (91.89\%) patients and nasopharyngeal swabs from two patients. Out of 36 samples sent for qPCR analysis, reports of only 11/36 (30.5\%) samples were available at the time of conclusion of this study. These results confirmed the presence of Influenza type A/H1N1pdm09 strains in 7 (63.63\%) patients. However, no significant difference was observed in the clinical features and complications of PCR positive and negative patients.

\section{Discussion}

Our study suggests that patients presenting with cough, nasal congestion, wheezing, and chest pain are likely to develop complications of respiratory tract infections such as pneumonia and should be suspected and investigated for influenza A (H1N1) infection. Furthermore, no difference in the severity of symptoms between PCR positive and PCR negative patients indicate that treatment may be considered in the lines of Influenza A infections by the attending physician in the light of current influenza epidemic. This is supported by previous studies which showed that clinical characteristics of patients presenting with ILI or SARI were significantly associated with the presence of influenza A(H1N1)pdm09 $[8,9]$.

In line with previous evidence [8,10-12], cough and fever were the cardinal symptoms of patients admitted to the pulmonology department. Similarly, abnormal radiological findings were reported in majority of patients in our study. Patchy infiltration, consolidations, and pleural effusions have also been reported previously [10]. Unlike other studies [11], our patients did not report diarrhoea. Occurrence of diarrhoea is common in H1N1, mainly in children and also in adults during pandemics. However, it is less commonly reported in seasonal influenza. Children are also more likely to develop complications of influenza irrespective of the severity of symptoms [11]. Our cohort did not include children, however a significant association of the development of ARDS with decreasing age reaffirms this notion.

Evidence of exposure or contact with sources of influenza virus was not reported by majority of patients in this study indicating that the primary source may have been from the animals to which humans are not resistant due to the absence of innate re-acquired immunity to the genetically re-assorted influenza virus. Animal and birds have their own set of influenza viruses that cause flu outbreaks in respective species. Although the swine flu virus (swine influenza A virus) is specie specific but zoonotic jumps have been seen crossing the specie barrier from pigs to humans [7]. In this new host, the virus undergoes genetic re-assortment leading to the origin of a new hybrid variety of super-virus to which humans lacks both innate and acquired immunity. This subsequently spreads swiftly in human population, posing a threat in the form of Influenza pandemic [7]. Index of suspicion of H1N1 increases with history of contact of patient with probable sources. Women, especially house wives, in Pakistani culture spend most of their time in the house and are therefore less frequently exposed to the

Table 2. Clinical parameters of admitted patients.

\begin{tabular}{lccc} 
& & Std. dev & P-value* \\
Variable & Mean & 0.58 & $<0.001$ \\
Temperature $^{\circ} \mathrm{C}(\mathrm{n}=37)$ & 38.75 & 6.83 & $<0.001$ \\
\hline Respiratory rate $($ per minute) $(\mathrm{n}=28)$ & 31.82 & 10.98 & $<0.001$ \\
\hline Oxygen saturation $\%(\mathrm{n}=33)$ & 81.09 & 8046 & 0.864 \\
\hline White cell count $(\mathrm{n}=19)$ & 11321 & \\
\hline
\end{tabular}

*One sample $t$-test: Std. dev: standard deviation.

Table 3. Binary logistic regression for the association of continuous predictors with the development of ARDS.

\begin{tabular}{lccc} 
Variable & Odds ratio & 95\% CI & p-value \\
Age (years) & 0.818 & $(0.5833,1.1476)$ & 0.055 \\
\hline Temperature (oC) & 0.0001 & $(0.0000,3242.1)$ & 0.166 \\
\hline Respiratory rate (per minute) & 0.989 & $(0.5220,1.8729)$ & 0.973 \\
\hline Oxygen saturation (\%) & 0.746 & $(0.4587,1.2144)$ & 0.053 \\
\hline White cell count & 1.001 & $(0.9996,1.0015)$ & 0.048 \\
\hline
\end{tabular}

Cl: confidence interval. 
sources of influenza unless a family member is infected. Surprisingly, majority of patients (67.5\%) presenting with SARI in our study were house wives and our data does not show any history of contact with infected patients. Furthermore, women going out of their houses wear veils which reduces their chance of acquiring an infection. This finding needs to be investigated in further studies.

Approximately $32 \%$ of our patients developed acute respiratory distress syndrome (ARDS) and subsequently needed mechanical ventilation. Patients who had severe nasal congestion and infiltration on chest radiograph were more likely to develop ARDS. Similarly decrease in oxygen saturation, younger age, and higher white cell count was significantly associated with the development of ARDS. Given the pathogenesis of ARDS, the association of these signs and symptoms with ARDS is expected [13], however presentation of patients with these findings will specifically help attending physician to plan aggressive management of patient prior to the development of ARDS.

No significant difference in the severity of patients' symptoms based on RT-PCR positivity were noted, which indicates that the initial suspicion at the time of admission should be the guiding point for the attending physician on the lines of H1N1 suspected case. This should however be interpreted with caution as we do not know if the patients presented, and their swabs obtained, within 5-7 days after the onset of Influenza like symptoms. Evidence suggests that PCR positivity is doubtful after 5-7 days of active infection [7].

Reported death of fewer patients (3 out of 37 patients) in this study compared to previous studies $[10,12]$ indicate that the use of antivirals and complementary antibiotics in ILI and SARI were effective and justified to reduce significant morbidity and mortality. Antiviral agents such as oral oseltamivir and inhaled zanamivir are associated with better disease outcomes, shorter duration of disease, and fewer rates of hospitalization in large meta-analyses [14]. However the quality of evidence available for these meta-analyses is limited and with low confidence of effect.

This study is not without limitations. As mentioned above, we did not have data regarding the duration between onset of ILI or SARI symptoms and presentation to the hospital. We also did not have RT-PCR reports for all patients limiting correlation of clinical signs and symptoms with confirmed presence of the virus.

In conclusion, clinical signs and symptoms of influenza like illness or severe acute respiratory illness significantly predict the development of complications irrespective of the positivity or negativity of laboratory PCR reports. It is recommended that these patients should be rigorously managed at presentation to prevent the development of complications and full blown respiratory disease.

\section{References}

1. Zambon MC. Epidemiology and pathogenesis of influenza. J Antimicrob Chemoth 1999;44(Suppl B):3-9.

2. Chan M. World now at the start of 2009 influenza pandemic. Available from: http//www.who.int/mediacentre/news/statements/2009/h1n1_ pandemic_phase6_20090611/en/

3. WHO. Influenza Update $\mathrm{N}^{\circ}$ 258. Accessed on: March 2016. Available from: http://www.who.int/influenza/surveillance_monitoring/updates/2016_03_07_surveillance_update_258.pdf?ua=1

4. WHO. Human infection with pandemic (H1N1) 2009 virus: updated interim WHO guidance on global surveillance. Available from: http://www.who.int/csr/resources/publications/swineflu/interim guidance/en/index.html

5. Hospital sends swine Faisalabad flu patient home. DAWN [Internet] 2009 Aug 14. Available from: http://www.dawn.com/news/ 935493

6. Ministry of National Health Services, Regulations and Coordination. Influenza Awareness Seminar, Islamabad: 4th February 2016.

7. Jafri SSI, Ilyas M, Idrees M. Swine flu: A threat to human health. Biotechnol Mol Biol 2010;5:46-50.

8. Nisar N, Aamir UB, Badar N, et al. Prediction of clinical factors associated with pandemic influenza A (H1N1) 2009 in Pakistan. PloS one 2014;9:e89178.

9. Badar N, Aamir UB, Mehmood MR, et al. Influenza virus surveillance in Pakistan during 2008-2011. PloS One 2013;8:e79959.

10. Karanji J, Gaude GS. Clinical characteristics and outcome of H1N1 (2009) pneumonia with special reference to radiological features in a tertiary care hospital in northern Karnataka. Al Ameen J Med Sci 2013;6:44-50.

11. Komiya N, Gu Y, Kamiya H, et al. Clinical features of cases of influenza A (H1N1) v in Osaka prefecture, Japan, May 2009. Eur Commun Dis Bull 2009;14:506-13.

12. Deng L-H, Zeng Y-L, Feng P, et al. Clinical characteristics of critical patients with pandemic influenza A (H1N1) virus infection in Chengdu, China. J Zhejiang Univ Sci B 2012;13:49-55.

13. Bellingan GJ. The pulmonary physician in critical care $\bullet$ : The pathogenesis of ALI/ARDS. Thorax 2002;57:540-6.

14. Hsu J, Santesso N, Mustafa R, et al. Antivirals for treatment of influenza: a systematic review and meta-analysis of observational studies. Ann Intern Med 2012;156 512-24. 